\title{
Adequate formalization
}

\author{
Michael Baumgartner • Timm Lampert
}

Received: 28 November 2006 / Accepted: 29 June 2007 / Published online: 18 August 2007

(C) Springer Science+Business Media B.V. 2007

\begin{abstract}
This article identifies problems with regard to providing criteria that regulate the matching of logical formulae and natural language. We then take on to solve these problems by defining a necessary and sufficient criterion of adequate formalization. On the basis of this criterion we argue that logic should not be seen as an ars iudicandi capable of evaluating the validity or invalidity of informal arguments, but as an ars explicandi that renders transparent the formal structure of informal reasoning.
\end{abstract}

Keywords Logic Philosophy of logic $\cdot$ Theory of formalization · Applied logic

\section{Introduction}

The problem of adequately transforming statements of natural language into the formalism of standard propositional or predicate logic is a problem most students of logic encounter without being presented with satisfactory solutions. ${ }^{1}$ Introductions to standard logic usually illustrate the formalization of natural language with a handful of paradigmatic examples supplemented with commentaries to the effect that formalization is essentially based on an artistic skill that cannot be regularized or taught methodically. Adequate formalization, according to the standard view, is a matter of experience and should thus not be a focus of a logician's interest. Nevertheless, formalizations are frequently used as a means to reconstruct arguments or to prove theorems, e.g. in metamathematics or mathematical logic. The adequacy of the adopted formalizations, however, is hardly ever substantiated, as this is seen as a matter of 1 Throughout this article we confine our discussions to first-order formalization. For the sake of simplicity,
we thus abstain from explicitly relativizing our definitions to first-order logic.

M. Baumgartner $(\varangle)$

Department of Philosophy, University of Bern, Laenggassstr. 49a, 3012 Bern, Switzerland

e-mail: baumgartner@philo.unibe.ch

T. Lampert $(\bowtie)$

Department of Philosophy, Baker Hall 135, Carnegie Mellon University, Pittsburgh, PA 15213, USA

e-mail: lampert@andrew.cmu.edu 
linguistic intuition or taken to be sufficiently legitimized by common practice. The widespread neglect to justify formalizations involved in proofs becomes unsatisfactory when it comes to choosing among alternative formalizations or to answering questions that arise when the effort to come up with arguments for or against given formalizations is actually made. Proofs involving the transformation of ordinary language to a formalism, such as validity proofs of ordinary language arguments or proofs of metamathematical theorems, are convincing only if they rely on a systematic understanding of the adequacy of the formalizations resorted to. ${ }^{2}$

The literature explicitly addressing the formalization of natural language can be broadly subdivided into two groups: On the one side, there are the authors claiming that formalizations of natural language should and can be generated by an effective procedure that takes sign sequences of colloquial language as inputs and produces adequate formalizations as outputs. On the other side, there is a group of authors which, rather than advancing formalization procedures, develop criteria (or guidelines) that are intended to determine (or help determine) whether a given formula is an adequate formal representation of a given natural language text. Davidson's theory of meaning, Chomsky's generative grammar and, most of all, Montague's universal grammar are the best known approaches to formalization that implicitly or explicitly subscribe to the ambitious project to define an effective formalization procedure (cf. e.g. Davidson 1984; Chomsky 1977; Montague and Thomason 1974). While Davidson contends himself with programmatic declarations to the effect that the availability of such a procedure is a precondition of an adequate theory of meaning, Chomsky and Montague have actually undertaken the effort to partially sketch the details of such a procedure. However, even though Chomsky refers to one of the outputs of his procedure as "logical forms" (LF), he clearly distinguishes a linguist's LFs from a logician's logical forms or formalizations. ${ }^{3}$ Montague, in turn, substantially constrains the class of colloquial texts which he claims to be tractable by his formalization procedure. For instance, the theory of language which he devises in Montague (1974b) involves a translation function that assigns expressions of an intensional formalism to textual arguments belonging to a disambiguated, i.e. non-natural, language. ${ }^{4}$ In contrast, while the fragment of English which he mechanically formalizes in Montague (1974a) features ambiguities, it only contains about three dozen basic expressions. All in all, thus, the project of developing an effective formalization procedure that would link natural languages and first-order logic is not even close to a successful completion. Moreover, that this project is successfully completable can be doubted in principle. Its completion would require solutions to intricate linguistic problems as, for instance, the context sensitivity of colloquial statements, which is far from being mechanically resolvable. Furthermore, the so-called misleading form thesis (cf. e.g. Brun 2004, ch. 7.1) raises principal philosophical objections against the claim that a statement's logical formalization, in one way or another, hinges on its surface grammar. Rather than on its grammatical surface, the formalization of a natural language utterance depends on the latter's informal interpretation, which is notoriously ambiguous and context dependent.

\footnotetext{
2 Here and throughout this paper two categories of proofs must be kept apart. The first category consists of proofs involving formalizations as (in)validity proofs of arguments and many mathematical and metamathematical proofs, and the second comprises the proofs that do not rest on formalizations as formal proofs by syntactic transformations of formulae. In Sect. 4 we shall show that the proofs of the first category can only be said to be proofs in a weak sense, i.e. proofs which formally explicate informal reasoning. They are not proofs in a strong sense according to which a proof is an evaluation of informal reasoning.

3 Cf. e.g. Chomsky (1986), p. 67, footnote 11: "The status and properties of LF are empirical matters, not to be settled in terms of considerations of valid inference and the like". For more details on the relationship between LFs and standard logical forms cf. e.g. Neale (1994).

${ }^{4}$ For an introduction to Montague's Universal Grammar cf. Link (1979).
} 
In this paper, thus, we do not presuppose that adequate formalizations can be mechanically generated from colloquial texts and, accordingly, we are not going to further discuss the efforts to define effective formalization procedures. ${ }^{5}$ Instead, we assume the starting point of logical formalization to consist in interpretations of natural language texts and focus on the literature concerned with criteria of adequate formalization that serve to justify or evaluate formalization attempts. There exists only a handful of studies, including Blau (1977), Epstein (1990, 1994), Sainsbury (1991), or recently and most thoroughly Brun (2004), that explicitly focuss on the design of formalization criteria. To this second, criteria-driven thread in the formalization literature Brun (2004), p. 269, refers as the traditional project of formalization. Within this project, the studies mentioned above devise what we call the traditional picture of logical formalization. None of the representatives of the traditional picture claim to offer sets of rules that would be severally necessary and jointly sufficient to resolve controversial mappings of logical formulae to statements. Rather, by reconstructing the theoretical underpinnings of the usual practice, they attempt to develop informal guidelines that shall help to clarify controversial formalizations. By identifying problems of the traditional picture we show that the usual practice is not a serviceable guide when it comes to defining consistent criteria of adequate formalization. We then go on to solve these problems by developing a necessary and sufficient criterion of adequate formalization which essentially rests on the insight that logical formalization cannot be separated from semantic analysis. We label this new approach within the traditional project the new picture of adequate formalization.

\section{The traditional picture}

In this section we discuss two main features of the traditional picture: criteria of correctness and surface maxims. We identify problems of a syntactic as well as of a semantic criterion of correctness and argue that customary surface maxims cannot serve as criteria of adequate formalization.

\subsection{Preliminaries}

Among the representatives of the traditional picture Blau (1977) and Brun (2004) most explicitly argue that formalizations of natural language texts are adequate if (I) they are correct, (II) they are maximally faithful to the syntactic surface of the respective text, and (III) they exhibit a unity of logical form, i.e. they are either equivalent or they represent the same form on various levels of specification. The first two determinants of the traditional picture prompt non-relational criteria of adequate formalization, while the third results in relational ones. We address the non-relational criteria of adequacy in this paper-Sects. 2.2 and 2.3, respectively — while (III) constitutes the focus of our article The Unity of Logical Form (unpublished). All three determinants of the traditional picture give rise to problems, some of which are acknowledged in the literature others are not. We argue that these defects are severe enough to replace the traditional by a new picture of logical formalization.

Before (I) and (II) are clarified, the object of formalization needs to be specified: It is usually presumed that logical formalization starts with a text $\mathcal{T}$ (a finite sequence of signs) which is analyzed to be composed of $A_{1}, \ldots, A_{n}$ such that $A_{1}, \ldots, A_{n}$ are statements capable of

\footnotetext{
${ }^{5}$ Even if the misleading form thesis is not taken to refute the procedure-driven approach to logical formalization, it is plain that the availability of formalization criteria is conceptually prior to a successful development of formalization procedures. Any such procedure must prove its strength by generating adequate formalizations, which adequacy has to be assessable with recourse to criteria.
} 
being true or false and $\mathcal{T}$ is interpretable as a truth-function of $A_{1}, \ldots, A_{n}$ only. We call such a truth-functional analysis of $\mathcal{T}$ an interpretation of $\mathcal{T}$. An interpretation can be expressed by means of a well-formed concatenation of $A_{1}, \ldots, A_{n}$ using truth-functional connectives, such that equivalent concatenations constitute the same interpretation. $A_{1}, \ldots, A_{n}$ may have identical truth conditions and may be identical in type, i.e. they may be representable by one and the same sentence. The truth-functional decomposition of $\mathcal{T}$ is not required to result from a syntactic analysis that fragments texts with respect to criteria such as full stops or syncategorematic phrases as "and", "or", "if ... then ..." etc. $\mathcal{T}$ may well be composed of questions or exclamations as e.g. the text $\mathcal{T}_{1}$ : "Is it raining? Yes! Then we take the umbrella". In this case, $\mathcal{T}_{1}$ might be interpreted as a truth-function of the two statements (i) "It is raining" and (ii) "We take the umbrella", such that $\mathcal{T}_{1}$ is true unless (i) is true and (ii) is false. Thus, the decomposition of a text into statements capable of being true or false is an interpretative task that is not a part of logical formalization. It is to be conducted prior to formalizing $\mathcal{T}$. The criteria of formalization available in the literature are usually confined to the formalization of single statements. We shall follow this practice in this section and take up the formalization of compound texts in the next.

\subsection{Correctness}

The correctness of a formalization is alternatively assessed with recourse to a syntactic or a semantic criterion of correctness. The syntactic criterion evaluates correctness based on a comparison of formal and informal inferential dependencies among formalizations and corresponding statements, and the semantic criterion compares truth conditions of statements and their formulae. The criteria of correctness shall be discussed in that order.

Syntactically, correctness of a formalization $\Phi$ of a statement $A$ is defined in terms of (COR): ${ }^{6}$

(COR) The formalization $\Phi$ of a statement $A$ is correct iff every inference $S$, such that the formalization $\Psi$ of $S$ contains $\Phi$ as a premise or a conclusion and $\Psi$ is formally valid, is informally valid.

More concisely put, (COR) stipulates

$$
\begin{aligned}
\Phi, \Gamma \vdash_{L} \Omega & \Rightarrow A, C \vdash_{I} B \text { and } \\
\Delta \vdash_{L} \Phi & \Rightarrow D \vdash_{I} A
\end{aligned}
$$

where $\Phi, \Omega$ are the formalizations of the statements $A$ and $B$, respectively, and $\Gamma, \Delta$ are sets of the formalizations of the statements contained in the sets $C$ and $D(\Gamma, \Delta, C, D$ may be empty), while $\vdash_{L}$ stands for formal validity and $\vdash_{I}$ represents informal validity.

An inference is valid iff, given the truth of its premises, its consequences cannot be false. Furthermore, while formal validity is to be understood on the basis of a respective calculus, an inference is said to be informally valid if it is judged to be valid without compulsory recourse to any criterion of its validity. Similarly, we often use expressions correctly without being able to define them or justify their application. In this sense, informal validity constitutes the unquestionable basis for formalizations of natural language. ${ }^{7}$

\footnotetext{
6 Cf. e.g. Blau (1977), p. 4, Brun (2004), p. 214.

7 Making the adequacy of formalizations dependent on the notion of informal validity has considerable consequences for the sense in which the validity or invalidity of colloquial arguments can be said to be provable. This problem and the question as to what contribution to the definition of criteria of adequate formalization can be expected from a concept as vague as informal validity will be addressed in Sect. 4 .
} 
(COR) is seen as a necessary condition of the adequacy of a formalization. It states that a formula assigned to a statement is inadequate if it allows for inferences that the statement does not sanction or if it follows from premises the corresponding statement does not informally follow from. According to (COR), formalizing any statement by $p$ along with a suitable realization is always correct. ${ }^{8}$ In case of non-tautologous and non-contradictory statements (COR) demands that realizations informally suit the formalized statement. This is illustrated by the following example:

(a) Shamus likes cheese.

$p$ : Winston likes ham.

While (1) is (COR)-correct for its own verbalization as induced by (2), i.e. for "Winston likes ham", (1) is not a correct formalization of (a), because $p$ is formally equivalent to $p$, whereas "Shamus likes cheese" is not informally equivalent to "Winston likes ham". (COR), thus, identifies (2) as an unsuitable realization with respect to a formalization of (a).

However, the application of (COR) faces a termination problem. Any formula implies an infinite number of other formulae. To see this, it suffices to consider $\vee$-introduction: $A \vdash A \vee B$. (COR) requires that it be verified for all of these inferences whether they have informal counterparts or not - a task that obviously cannot be completed. That means whether a formalization is correct in terms of (COR) can only be falsified. Moreover, as Brun (2004), p. 217, points out this termination problem induces yet another difficulty for the application of the correctness criterion. Suppose we are to evaluate the correctness of a formula $\Phi$ assigned to a statement $A$. In order to do so, we are to check whether, say, $\Phi \vdash_{L} \Omega$, which we assume to be (formally) valid, has an informal counterpart. This apparently presupposes that $\Omega$ is assigned to another statement $B$ such that we are then able to evaluate whether $B$ in fact informally follows from $A$, which, in turn, presupposes the correctness of formalizing $B$ by $\Omega$. Therefore, the evaluation of the correctness of a single formalization $\Phi$ is not independent of the correctness of other formalizations. This, again, presupposes that $\Phi$ is a correct formalization of $A$. Thus, the application of (COR) gives rise to a circularity problem.

The fact that the correctness and, hence, the adequacy of the formalizations of different statements are interdependent might not be seen as problematic to somebody maintaining a holistic view of logical formalization as e.g. Davidson (1980), pp. 139-140, who claims that the adequacy of all formalizations of all statements of a language must be assessed as a whole. According to such an account, no formalization is ever unquestionably established to be correct. Every seemingly correct formalization constantly risks to be identified as incorrect in light of other formalizations. Thus, whoever adopts such a holistic view no longer considers (COR) to be a criterion of formalization which determines for each formalization candidate whether it correctly captures a given statement or not. Within a holistic framework, (COR) becomes a mere rule of thumb. As indicated in the introduction, however, we are here concerned with criteria of adequate formalization, not with rules of thumb. If (COR) is taken to be a formalization criterion, the mutual interdependence of the correctness of manifold formalizations is highly problematic as, due to that interdependence, (COR) is affected by the circularity problem.

8 Cf. Blau (1977), p. 7, and Brun (2004), p. 210 et sqq., 240-241. The notion of a realization is here being used along the lines of Epstein (1994), p. 13, i.e. in terms of an assignment of expressions of natural language to the categorematic parts of a formula. The categorematic expressions contained in a formula are its propositional variables, proper names and predicate letters. In the literature, terms as "correspondence scheme", "scheme of abbreviation", "dictionary" or "key" are used synonymously with "realization" (for details cf. Brun (2004), p. 140 , footnote 3 ). 
Yet, as mentioned above, correctness does not necessarily have to be spelled out by syntactic criteria, it can be assessed semantically as well. The basic idea behind a semantic criterion of correctness can be concisely put, its detailed formulation, however-as will be shown below-is more intricate. Semantically, a formalization $\Phi$ of a statement $A$ is said to be correct iff $\Phi$ and $A$ have the same conditions of truth and falsehood. Postponing the question as to how exactly conditions of truth and falsehood of formulae and statements can be compared, it is worth to point out that an application of a semantic criterion of correctness is free from circularities. Contrary to (COR), assessing the correctness of a formalization $\Phi$ on semantic grounds does not presuppose the correctness of other formalizations. Nonetheless, as (COR), a semantic criterion of correctness is affected by a termination problem. Formal semantics of first-order logic generates an infinite amount of interpretations for a formula, based on which truth and falsehood of that formula are evaluated. Therefore, as long as the application of the semantic criterion does not yield a discrepancy between truth conditions of a statement and its formalization, the correctness of that formalization is not determinable - the application of the criterion simply does not terminate.

Contrary to the (COR)-variant of the termination problem, however, there are proposals to secure the termination of a semantic assessment of correctness available in the literature. Blau (1977) and Brun (2004) attempt to evade the semantic variant of the termination problem by stipulating that evaluating the correctness of a formalization by means of semantic criteria does not require balancing all truth conditions of a statement and its formalization separately. It suffices to match general descriptions of suitable classes of interpretations. Brun (2004), p. 211, illustrates what this means by the following example:

(b) All animals have a head.

$$
\forall x(F x \rightarrow G x)
$$

$$
F: \ldots \text { is an animal; } G: \ldots \text { has a head }
$$

The models of (3) and the circumstances in which (b) is true can be described structurally: The extension of $F$ is a subset of the extension of $G(\Im(F) \subseteq \Im(G))$, i.e. animals constitute a subset of the headed objects. (3) and (b) are false iff there is at least one object contained in the extension of $F$ but not of $G(\mathfrak{I}(F) \nsubseteq \mathfrak{I}(G))$, i.e. iff there is at least one unheaded animal. If the conditions of truth and falsehood of (3) and (b) coincide with respect to these structural descriptions - thus Brun's proposal to solve the termination problem-the application of the semantic criterion terminates and attests the correctness of (3). ${ }^{9}$

Prima facie, thus, the prospects of the semantic criterion of correctness seem to be very promising. However, as anticipated above, a closer look at the details of this criterion reveals that it faces a serious problem of its own. Put briefly, in order to guarantee the comparability of the truth conditions of formulae and statements and to ensure the equivalence of the syntactic and the semantic criterion, the interpretative space of formal semantics must be confined in a way that seriously endangers the whole endeavor of logical formalization. As will be substantiated promptly, the core of this problem stems from the fact that according to the traditional picture not all interpretations of formulae can be evaluated informally, but only suitable ones. Let us hence discuss the details of a semantic notion of correctness. Ordinarily, it is spelled out as follows: ${ }^{10}$

\footnotetext{
9 Brun does not offer a procedure or a criterion that identifies the structural descriptions of truth conditions. Generating these descriptions is left to informal intuition. In Lampert (2006) a mechanical procedure is developed for first-order logic that, given a formula $\Phi$, construes structural descriptions of the models and counter-models of $\Phi$ in a finite number of steps. By applying this procedure and paraphrasing the resulting structural descriptions the solution of this termination problem can be completed.
}

10 Cf. e.g. Brun (2004), p. 210. 
(TC) The formalization $\Phi$ of a statement $A$ is correct iff relative to all suitable interpretations $\mathfrak{\Im}_{S}$ of $\Phi, \Phi$ has the same conditions of truth and falsehood as $A$ has according to the informal judgement.

The application of (TC) presupposes that it is determined what truth and falsehood conditions of formulae are as opposed to truth and falsehood conditions of statements and how these conditions can be compared. According to (TC), this comparability shall be guaranteed by suitable interpretations. Thus, two notions involved in (TC) call for clarifications: suitable interpretation and sameness of truth and falsehood conditions.

Suitable interpretations of a formula for Blau (1977) and Brun (2004) are interpretations of the propositional variables, of the proper names and of the predicate letters that are limited in extent due to informal inferential dependencies among the natural language expressions that are assigned to the categorematic parts of a formula by a given realization. The sort of limitation involved in suitable interpretations is best clarified by concrete examples. First, consider statement (c) along with its forthright formalization (5) and realization (6).

(c) Cameron is a mother or Cameron is a woman.

$$
p \vee q
$$

$p$ : Cameron is a mother.; $q$ : Cameron is a woman.

The set of suitable interpretations of (5) does not contain 4 elements as would be generated by formal semantics, but only 3 -more precisely, instead of $\Im(p)=T, \Im(q)=T ; \Im(p)=$ $T, \Im(q)=F ; \Im(p)=F, \Im(q)=T ; \Im(p)=F, \Im(q)=F$, it only comprises the following 3 interpretations: $\Im(p)=T, \Im(q)=T ; \Im(p)=F, \Im(q)=T ; \Im(p)=F, \Im(q)=F$. On informal grounds, it is excluded that something is a mother but no woman. This informal judgement, accordingly, eliminates $\Im(p)=T, \Im(q)=F$ from the set of suitable interpretations of (5). It is this elimination that allows for comparing the truth conditions of (5) and (c) in the first place. Were $\Im(p)=T, \Im(q)=F$ not discarded, formal semantics would confront us with a configuration that informally could not be judged coherently, because from Cameron's motherhood the truth of (c) follows, whereas Cameron not being a woman implies the falsehood of (c). Consequently, (TC) would be inapplicable. Hence, suitable interpretations result from ordinary interpretations by limiting the interpretative space of formal semantics due to informal inferential dependencies. ${ }^{11}$ This constraint is warranted-thus the standard arguments in the literature-because truth conditions of statements and formulae are guaranteed to be comparable only within limited interpretative spaces. ${ }^{12}$

The truth conditions of a formalization $\Phi$ of a statement $A$ consist in the models of $\Phi$ among its suitable interpretations, i.e. in its suitable models. Thereagainst, the falsehood conditions of $\Phi$ consist in its suitable counter-models. The truth and falsehood conditions of $A$, on the other hand, are generated by paraphrasing the suitable interpretations relative to a given realization of $\Phi$ and informally assessing the truth values of $A$ with recourse to these paraphrases. For instance, the paraphrases of the suitable interpretations of (5) in virtue of its realization (6) are the following: (i) "Cameron is a mother", "Cameron is a woman"; (ii)

\footnotetext{
11 Sainsbury (1991), p. 64, restricts the interpretative space of formal semantics even further: "A necessary condition for adequacy is that every sentence in the formalization should be true (false) on an intended interpretation iff the corresponding English sentence is true (false)". For propositional logic Sainsbury defines an intended interpretation to be an interpretation "which assigns to the relevant sentence-letters the same truth values as the ones the corresponding English sentences possess".

12 In a very similar vein, Quine (1953), p. 439, takes one of the most conspicuous characteristics of a natural language to be its truth value gaps which, according to him, must be ignored upon formalizing natural language.
} 
"Cameron is no mother", "Cameron is a woman"; (iii) "Cameron is no mother", "Cameron is no woman". Under conditions (i) and (ii) the statement (c) is informally judged to be true. Thus, the truth conditions of (c) are (i) and (ii). Whereas (iii) is the falsehood condition of (c), because when Cameron is neither a mother nor a woman, (c) is informally judged to be false. A comparison of the truth and falsehood conditions of a statement $A$ and its formalization $\Phi$ amounts to confronting the suitable models and counter-models of $\Phi$ with those paraphrases of the suitable interpretations that are informally seen to render $A$ true and false, respectively. The models and counter-models are evaluated by formal semantics, their paraphrases are judged informally. (TC) - by stipulating sameness of truth and falsehood conditions of $\Phi$ and $A$-demands that the informal judgement determines the paraphrases of suitable models and counter-models to render the formalized statement true and false, respectively.

However, limiting the interpretative space of formal semantics ${ }^{13}$ in this vein gives rise to a serious problem. (TC) is not embedded in classical semantics of first-order logic, because when (TC) is applied, interpretations $\mathfrak{\Im}$ of a formula $\Phi$ can be discarded. That means (TC) induces a revision of the semantics of logical formulae to the effect that the latter no longer mean what they mean according to classical semantics. This revision changes the whole logic of first-order formulae. For instance, if the interpretation $\Im(p)=F, \Im(q)=F$ of the formula $p \vee q$ is eliminated from the set of suitable interpretations, $p \vee q$ is interpreted in terms of a tautology, which obviously it is not according to propositional logic. Thus, strictly speaking, (TC) is no criterion that regulates formalization within first-order logic. It cannot even be claimed that (TC) regulates formalization within any logic, because the semantics of the formulae are no longer taken at face value. Using a logical notation while selectively adapting its interpretation amounts to confusing formal and informal reasoning and reduces logical notation to stenography. Semantic criteria of adequate formalization must draw on the semantics of their target logic as a whole — one cannot restrict semantics in order to obtain adequate formalizations. When formalizing within some logic $\mathbf{L}$, the latter's semantics needs to be presupposed without restriction. If the semantics of $\mathbf{L}$ prohibits adequate formalizations of a text $\mathcal{T}, \mathcal{T}$ simply cannot be formalized within $\mathbf{L} .{ }^{14}$ Thus, (TC) suffers from the problem of suitable interpretations.

This problem arises whenever the semantic criterion of correctness is intended to be equivalent to (COR). In order to see this, reconsider statement (c), "Cameron is a mother or Cameron is a woman", and its formalization (5), $p \vee q$, with realization (6), which is correct according to (COR) and (TC). Yet, if $\Im(p)=T, \Im(q)=F$ were not discarded, (5) would no longer have the same truth conditions as (c). $\Im(p)=T, \mathfrak{s}(q)=F$ renders (5) true, whereas Cameron being a mother and no woman is no meaningful truth condition of (c), because a corresponding informal judgement would be incoherent (see p. 8). Thus, if the comparison of truth conditions as induced by (TC) were not limited to suitable interpretations, the semantic criterion of correctness would not be equivalent to the syntactic criterion (COR).

The problem of suitable interpretations shall be postponed for now. We come back to it below and propose a solution for it. For the time being, the traditional picture needs to

\footnotetext{
13 It might be objected that the notion of a suitable interpretation, strictly speaking, does not limit the interpretative space of formal semantics, but merely determines that certain interpretations generated by formal semantics must be ignored when it comes to comparing truth conditions of statements and formulae. However, whether such a comparison is said to require that certain interpretations be eliminated or merely ignored is of terminological relevance at best. Limiting the interpretative space of formal semantics may, thus, alternatively be understood in terms of eliminating or of ignoring unsuitable interpretations.

14 Such a view is implicitly endorsed by Strawson (1952), ch. 6. Strawson is not ready to ignore truth value gaps when formalizing natural language and, consequently, identifies a host of statements which he claims not to be adequately formalizable in first-order logic.
} 
be complemented by introducing the second non-relational constraint (cf. p. 3) ordinarily imposed on adequate formalizations: adherence to the syntax of formalized texts.

\subsection{Surface maxims}

In the literature, criteria of correctness are usually complemented by maxims which determine the adequacy of a formalization to not only hinge on its capturing the informally judged truth and falsehood conditions of a statement, but which moreover demand to do justice to the syntax of that statement. The additional maxims demanding syntax adherence are induced by the problem of trivialization. In regards to formalizing arguments this problem can be illustrated by what Blau (1977), pp. 17-18, calls unscrupulous formalizing. Consider argument (d) along with its formalization (7) and realization (8).

(d) Cameron is a mother. Therefore, Cameron is a woman.

$$
\begin{gathered}
F a \wedge G a \rightarrow G a \\
a: \text { Cameron; } F: \text {...is a mother; } \quad G: \text {...is a woman }
\end{gathered}
$$

First of all, note that representatives of the traditional picture often formalize arguments by means of argument schemes involving operators as " - " or " $\therefore$." that belong to metalanguage. Within first-order object language, however, the informal validity of arguments can only be captured in terms of formal implications. As we are exclusively concerned with first-order formalizations in the present context, we dispense with metalingual operators in formalization candidates throughout this paper. ${ }^{15}$ Against this background, (7) and (8) constitute a formalization that captures the premise and the conclusion of (d) as well as the whole argument in a both syntactically and semantically correct way. Unscrupulously formalizing an informally valid argument, thus, amounts to conjunctively adding a formula representing the conclusion to the formula(e) assigned to the premises. ${ }^{16}$

Trivializations of formalization efforts can even be aggravated. For instance, (COR) and (TC) allow for assigning any formal tautology to an informally tautologous statement as "It is raining or it is not raining". Clearly however, only a very specific subset of formal tautologies can be considered to adequately represent that statement. Were the adequacy of a formalization only dependent on (COR) and (TC), formalizing natural language would amount to an utterly trivial and non-illuminative endeavor. Not only is it compatible with (COR) and (TC) to assign an arbitrary tautology to any informally valid argument, all informally valid arguments can be assigned to one and the same tautology. Moreover, informally equivalent statements as

(e) Not all martians are green.

(f) Some martians are not green.

can generally be (COR)- and (TC)-correctly formalized by identical formulae, irrespective of whether their syntactic differences might be accountable for in the corresponding formalism or not. Thus, formalizing both (e) and (f) by $\neg \forall x(F x \rightarrow G x)$, given a suitable realization, fully conforms to (COR) and (TC). Yet, formalizing (e) and (f) by one and the same formula $\Phi$ reduces the formal proof of their equivalence to proving $\Phi \leftrightarrow \Phi$ and, hence, completely

15 This restriction to the first-order syntax is explicitly advanced as a possible formalization strategy by representatives of the traditional picture as Epstein (1990), p. 24, or Brun (2004), pp. 46, 243.

16 If it is presumed that an argument is formalized correctly only if all its component statements are formalized correctly, this trivialization problem merely affects arguments involving one single premise (cf. Brun (2004), p. 240 et sqq., for a detailed discussion of the trivialization problem). 
trivializes it. Considering (COR) and (TC) sufficient to identify adequate formalizations would trivialize every equivalence proof.

Surface maxims are thus introduced in order to identify adequate formulae given sets of formalizations that equally conform to (COR) and (TC). For instance, there are some obvious syntactic differences between (e) and (f). (e) mentions something that not every martian complies with, whereas (f) talks of some martians. Accordingly, in addition to compliance with (COR) and (TC) it is stipulated that the syntactic features of these statements must be represented by formulae adequately assigned to them. Though the formula $\neg \forall x(F x \rightarrow G x)$ mirrors the syntax of (e), its syntactic features do not have a counterpart in (f). Surface maxims are designed thus that they determine the aforementioned formula to adequately represent (e), but not (f).

Basically there are two directions available with regard to explicating how formulae represent syntactic features of a statement: (I) A syntactic feature of a formula must have a counterpart in the statement; (II) a syntactic feature of a statement must have a counterpart in the formula. Both of these directions can be found in formalization strategies available in the literature. While Brun's (2004) surface maxims are oriented in terms of (I), ${ }^{17}$ Epstein (1994) formalizes by abstracting from the content of statements in a stepwise manner and, thus, adopts strategy (II). Neither of these orientations of surface maxims yields necessary or sufficient criteria of adequate formalization. Take, for instance, formula (9):

(g) Men are mortal. Shamus is a man. Therefore, Shamus is mortal.

$$
\begin{aligned}
& \forall x(F x\rightarrow G x) \wedge F a \rightarrow G a \\
& a: \text { Shamus; } F: \text {...is a man; } G: \text {...is mortal }
\end{aligned}
$$

The formalization of the first premise of (9) is generally seen as an adequate formalization of "Men are mortal". Yet, it features a universal quantifier and a conditional, neither of which has a counterpart in (g). While a syntactic maxim in the vein of (I) may well distinguish between formulae adequately ascribed to (e) and (f), a maxim oriented in terms of (II) would misleadingly dictate to assign different formulae to:

(h) This conclusion is invalid.

(i) This conclusion is not valid.

The syntactic difference between (h) and (i) must not be captured by a difference in the formula adequately assigned to these statements. Furthermore, it would be inaccurate to predicate inadequacy of formalizations of statements containing an "if and only if" simply because the formalism resorted to does not feature the connective " $\leftrightarrow$ ". Surface maxims cannot be used as necessary or sufficient conditions for the adequacy of formalizations. They cannot be applied unconditionally such that they always pick one formula whose paraphrase is syntactically most similar to the corresponding statement. Their application must be relativized to a formalism and classes of equally correct formulae available as formalization candidates (cf. Sect. 3.2).

\section{The new picture}

This section introduces the idea of a complete formalization by first discussing the syntactic completeness criterion available in the literature. Completeness is commonly not seen as a

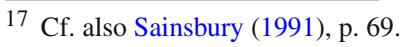


requirement adequate formalizations have to meet, because the version of this criterion provided in the literature not only suffers from a syntactic but also from a semantic termination problem. In this section we want to show that completeness has been discarded prematurely. Not only can a completeness criterion be devised in an operationalizable way, it moreover paves the way to solutions of the difficulties faced by the traditional picture. To this end, we first syntactically define completeness in a way that no longer gives rise to a semantic termination problem. Analogously to correctness, however, the application of our syntactic variant of completeness does not terminate. In consequence, we then go on to introduce a criterion which semantically captures both completeness and correctness and which we claim to be immune to all problems of the traditional criteria.

\subsection{Completeness}

The literature provides sketches of an additional adequacy criterion. Blau (1977) and Brun (2004) consider a syntactic criterion of completeness, yet discard it as necessary condition of adequate formalization because they take it to be too strong and to lack applicability. Spelling out what completeness of a formalization amounts to requires some conceptual preliminaries, because the existing version of this criterion crucially rests on a specialized notion of informal validity: informal formal validity. In order to clarify that notion, consider again the $\operatorname{arguments}(\mathrm{g})$ and $(\mathrm{d})$ :

(g) Men are mortal. Shamus is a man. Therefore, Shamus is mortal.

(d) Cameron is a mother. Therefore, Cameron is a woman.

(g) and (d) shall be taken to be valid on informal grounds. According to a prevalent view, statements have certain consequences merely due to their form and others due to the meaning of their constituents. ${ }^{18}$ The former are consequences based on informally formally valid arguments, while the latter result from informally materially valid arguments. ${ }^{19}$ This difference is illustrated by $(\mathrm{g})$ and $(\mathrm{d})$. While $(\mathrm{g})$ is commonly held to be valid on account of its plain form or syntax, it takes conceptual analysis of the notion of a mother to justify the informal validity of (d).

With recourse to the notion of informal formal validity completeness of a formalization $\Phi$ of a statement $A$ can be defined as follows:

(COM) The formalization $\Phi$ of a statement $A$ is complete iff every formalization $\Psi$, such that $\Psi$ is the formalization of an inference $S$ and contains $\Phi$ as a premise or a conclusion and $S$ is informally formally valid, is formally valid.

More concisely put, (COM) stipulates

$$
\begin{aligned}
A, C \vdash_{I f} B & \Rightarrow \Phi, \Gamma \vdash_{L} \Omega \text { and } \\
D \vdash_{I f} A & \Rightarrow \Delta \vdash_{L} \Phi
\end{aligned}
$$

where $\Phi, \Omega$ are the formalizations of the statements $A$ and $B$, respectively, and $\Gamma, \Delta$ are sets of the formalizations of the statements contained in the sets $C$ and $D$ ( $\Gamma, \Delta, C, D$ may be empty), while $\vdash_{L}$ stands for formal validity and $\vdash_{\text {If }}$ represents informal formal validity.

As we have seen in Sect. 2.2, formalizing any statement by $p$ is always correct. Yet, many formalizations that are commonly viewed as adequate illustrate that the adequacy of formalizations is often judged with recourse to further criteria than merely correctness and

18 Cf. Davidson (1967), Epstein (1990), Brun (2004).

19 For this distinction see Brun (2004), pp. 37-40 and pp. 327-334. 
surface similarity. To mention only a few well known formalization efforts that exemplify this practice: The formalization of Aristotelian syllogisms, Russell's analysis of definite descriptions or Davidson's account of action sentences were all motivated by the urge to formally represent informally valid arguments. Common practice, hence, not only aims for correct formalizations, but, furthermore, sometimes strives for a formal representation of the inner structure of a statement. By demanding completeness of a formalization, as expressed in $(\mathrm{COM})$, it is stipulated that $p$ is not necessarily adequate for any statement. As per (COM), it might be necessary to exhibit the inner structure of a statement in order to formally reproduce its informal consequences.

However, while completeness might in fact be an implicit motive behind many actual formalization efforts, completeness as expressed in (COM) is seriously defective. A first deficiency stems from the latter's dependency on the notion of informal formal validity. The distinction between informally formally and informally materially valid arguments is by far not as clear-cut as (g) and (d) prima facie might suggest. For in what sense can (g) be said to be informally formally valid, while (d) can only claim informal material validity? As anticipated above, answering this question with reference to the logical forms of (g) and (d) suggests itself: $(\mathrm{g})$ is valid in virtue of its form, while the validity of (d) stems from its semantic features. Yet, what is the argumentative backing of this stipulation? How are logical forms of statements identified? The syntax of natural language is ambiguous to the extent that logical forms of statements and arguments cannot be identified based on syntactic or grammatical criteria. The literature abounds with examples that illustrate this deficiency of natural language ${ }^{20}$ Consider, for instance, statements as "Horses are animals" whose commonly assigned logical form involves universal quantifiers and conditionals, both of which are lacking in the statement. Or take adjectival modification which sometimes corresponds to logical forms comprising conjunctions. The only way to identify the logical form of a statement is to assign a formula to that statement according to criteria of adequate formalization. ${ }^{21}$

That means explicating the difference between $(\mathrm{g})$ and $(\mathrm{d})$ with reference to their logical forms amounts to - consonantly with the usual practice - advancing the formal implication (11) as adequate formalization of (g), whereas the material implication (12) is considered to be adequate for $(\mathrm{d})$ :

$$
\begin{gathered}
\forall x(F x \rightarrow G x) \wedge F a \rightarrow G a \\
F a \rightarrow G a .
\end{gathered}
$$

Ascribing a universally quantified conditional to (g)'s premise illustrates that the formalization of a statement may well introduce syntactic features that do not have a correspondent in the statement. This finding, in turn, raises doubts as to whether (12) in fact constitutes the logical form of (d). These doubts are even intensified in light of examples as Davidson's formalization of action sentences which is commonly considered to be perfectly adequate:

(j) Cameron strolls through Bologna. Therefore, there is a stroll.

(j), according to Davidson's (1967) proposal, is adequately formalized as follows:

$$
\exists x(F x \wedge G x a \wedge H x b) \rightarrow \exists x F x
$$

The premise of ( $\mathrm{j})$ is, hence, rendered explicit as "There is a stroll and it is conducted by Cameron and it takes place in Bologna". (j) and (13) illustrate that adequate formalizations

20 Cf. Sainsbury (1991), pp. 339-340.

21 We take an in-depth look at how logical forms of natural language texts are identified in Lampert and Baumgartner (unpublished). 
sometimes introduce predicates that do not occur in the formalized statement. Correspondingly, (d) might just as well be analyzed in terms of the formal implication (14) rather than (12).

$$
G a \wedge H a \rightarrow G a,
$$

where $G$ represents "... is a woman" and $H$ stands for "... has a child". Thus $F$, which represents "... is a mother", is analyzed as a conjunction of $G$ and $H$. While attributing (12) to (d) yields the latter's validity to be of material nature, according to the conceptual analysis applied in (14), (d) would have to be seen as informally formally valid after all. If (13) is accepted as an adequate formalization of ( $\mathrm{j}$ ) and as showing that (j) is formally valid, one cannot reject (14) as an adequate formalization of (d) and hold that (d) is not formally valid. ${ }^{22}$ Conceptual analysis is an integral part of formalizing natural language. There is no clear demarcation line for the amount of conceptual analysis used in formalizing natural language. Furthermore, the question as to the formal or material nature of (d)'s validity can only be settled upon determining which of (12) and (14) constitutes an adequate formalization of (d). All of this shows that spelling out the difference between informal formal and informal material validity presupposes clarity about adequate formalization. The latter notion is more basic than the notion of logical form and more basic than the conceptual distinction between informal formal and informal material validity. Accordingly, recurring to this distinction in a criterion of adequate formalization as $(\mathrm{COM})$ renders the latter fundamentally circular.

In order to secure the criterion of completeness against these circularities the difference between informal formal and informal material validity must be dropped as done by Wittgenstein. For Wittgenstein there is no validity other than logical validity. ${ }^{23}$ Whatever is informally valid must be shown to be valid on formal grounds by means of logical formalization involving conceptual analysis. Every informally valid argument is to be formalized by a formal implication. In particular, (12) is not an adequate formalization of (d). In the following we adopt Sainsbury's label Tractarian view to denote that position. ${ }^{24}$

There are three main reasons commonly put forward as to why (COM) is not based on the Tractarian view and is, thus, specified with recourse to the formal-material distinction among informally valid inferences. A first objection against the Tractarian view is motivated by the urge to keep the task of formalizing natural language as free from semantic detours as possible. Yet, as the foregoing reasoning has shown, this demand cannot be fulfilled without circularities. Second, it is argued that statements involving color exclusion cannot, in principle, be formally captured. ${ }^{25}$ That means subject to this line of argument there is no formal contradiction that could be seen as an adequate formalization of an informally contradictory statement as "This spot is red and green at the same time". However, this criticism of the Tractarian view does not take into account that there may be a previously unthought of analysis of color predicates or new formalisms that are especially suited for statements involving color exclusion. ${ }^{26}$ Even if no such custom-built analysis could be found, that would not count against the Tractarian view, but rather against the first-order formalizability of statements involving color exclusion. The third objection against the Tractarian view is much more intricate. It is claimed that the Tractarian view jeopardizes the termination of logical formal-

\footnotetext{
22 Jackson (2007) casts doubts on the maintainability of the distinction between informal formal and informal material validity that are related to ours.

23 Cf. Wittgenstein (1995), 6.375.

24 Cf. Sainsbury (1991), 1st ed. ch. 6.2.

25 Cf. Read (1994), p. 251.

26 For a detailed discussion cf. Lampert (2000).
} 
ization. For instance, take argument (d) above. It is highly dubitable whether a finite formula assigned to a statement as "Cameron is a mother" could ever capture all the inferences as "Cameron is a woman", "Cameron has a child", "Cameron is a human being" etc. that can informally be drawn from that statement - as would be required by a Tractarian reading of (COM). Due to the vagueness of the concept of informal validity it is unclear whether it does even make sense to speak of a complete formalization that captures all the possible inferences that might be drawn from "Cameron is a mother". For brevity, call the problem of determining the informally valid inferences that have to be captured by an adequate formalization the termination problem of (COM).

There is a semantic and a syntactic aspect to (COM)'s termination problem. As indicated above, an unmanageable amount of inferences can be drawn from any colloquial statement due to its semantic implications. Yet, even if the distinction between informal formal and informal material validity could be accounted for somehow, "Cameron is a mother" would still have an infinite amount of informal (formal) consequences-it would e.g. imply infinitely many disjunctions containing "Cameron is a mother" as one disjunct. Hence, even the non-Tractarian reading of (COM) faces a termination problem.

In the literature, the semantic termination problem of (COM) is normally advanced as sufficient grounds on which to deny (COM) the status of a necessary condition of adequate formalization. Contrary to correctness, completeness is merely seen as a sometimes helpful guideline to be applied in a context-dependent way. ${ }^{27}$ However, abandoning completeness as necessary condition of adequacy is uncalled for. If completeness is not assessed for formalizations of singular statements, but for formalizations $\Phi_{1}, \ldots, \Phi_{n}$ of statements $A_{1}, \ldots, A_{n}$ such that a formalized text $\mathcal{T}$ is a truth-function of $A_{1}, \ldots, A_{n}$ (cf. p. 4), the class of inferences to be considered in order to determine the completeness of formalizations is limited by the decomposition of $\mathcal{T}$ into $A_{1}, \ldots, A_{n}$. A complete formalization only has to represent the dependencies among the component statements of $\mathcal{T}$. The main reason why statements are formalized in the first place is to transparently represent these dependencies. A thus contextualized completeness criterion does not face a semantic termination problem, because only premises and conclusions of inferences have to be considered that have truth conditions expressible within the finite vocabulary of the realizations of $\Phi_{1}, \ldots, \Phi_{n}$. As in case of (COR), however, a syntactic termination problem remains, for even with a finite vocabulary an infinite number of inferences can be formulated. Completely assuring the termination of both a correctness and a completeness criterion is - as will be substantiated below-only possible against the background of semantic variants of these criteria along with an account of how conditions of truth and falsehood are described structurally.

$\left(\mathrm{COM}^{\prime}\right)$ The correct ${ }^{28}$ formalizations $\Phi_{1}, \ldots, \Phi_{n}$ of $A_{1}, \ldots, A_{n}$ are complete iff every formalization $\Psi$, such that $\Psi$ is the formalization of an inference $S$, that does not consist of premises or conclusions other than $A_{1}, \ldots, A_{n}$ or their negations or of verbalizations of formulae exclusively composed of the categorematic expressions mentioned in the realizations of $\Phi_{1}, \ldots, \Phi_{n}$, and $S$ is informally valid, is formally valid.

\footnotetext{
27 Cf. Blau (1977), pp. 2-3 and pp. 16-17.

28 As correctness has already been established as a necessary condition for the adequacy of a formalization, not all formalization candidates of $A_{1}, \ldots, A_{n}$ need to be tested for completeness, but only the correct ones. Furthermore, as shown on p. 97 above, demanding (COR)-correctness of complete formalizations guarantees that the realizations of $\Phi_{1}, \ldots, \Phi_{n}$ suit $A_{1}, \ldots, A_{n}$. It is plain that subject to this integration of correctness into $\left(\mathrm{COM}^{\prime}\right)$ the latter inherits all of the former's problems. For the sake of spelling out completeness by syntactic criteria we are going to disregard these problems for the moment.
} 
$\left(\mathrm{COM}^{\prime}\right)$ limits the informally valid inferences to be captured by a complete formalization to the inferences involving the statements $A_{1}, \ldots, A_{n}$ and the vocabulary resorted to in the realizations of $\Phi_{1}, \ldots, \Phi_{n}$. Inferences expressible with the categorematic elements of $\Phi_{1}, \ldots, \Phi_{n}$ have to be considered in order to forestall unscrupulous formalizations. As to $\left(\mathrm{COM}^{\prime}\right.$ ), neither $\mathrm{Fa} \rightarrow G a$ nor $F a \wedge G a \rightarrow G a$, where $F$ stands for ". . . is a mother", $G$ for "... is a woman" and $a$ for Cameron, are complete formalizations of (d). Neither does justice to the informal dependency between "Cameron is a mother" and "Cameron is a woman". To see this, consider the formalization $F a \wedge G a \rightarrow G a$. Fa and Ga are two formulae composed of categorematic expressions contained in the realization of that formalization. According to that realization, $F a$ and $G a$ are to be verbalized in terms of "Cameron is a mother" and "Cameron is a woman", respectively. While $G a$ does not follow from $F a$, "Cameron is a woman" does follow from "Cameron is a mother". Furthermore, "Cameron is a mother" is informally equivalent to "Cameron is a mother and a woman", whereas $F a$ is not equivalent to $F a \wedge G a$. While (7) is thus determined to be incomplete by $\left(\mathrm{COM}^{\prime}\right),(14)$, i.e. $G a \wedge H a \rightarrow G a$ where $G$ stands for ". . is a woman", $H$ for ". . . has a child" and $a$ for Cameron, is not identified as incomplete by $\left(\mathrm{COM}^{\prime}\right)$ : (14) not only adequately captures the informal dependencies among the component statements of (d), but also all the inferences expressible with the vocabulary resorted to in (14). A complete formalization as (14) can thus be said to be maximally transparent with respect to informal dependencies among component statements and elements of its realization. All informal dependencies are explicitly formally represented, none are left implicit. That means the expressions assigned to the categorematic parts of a formula by its realization are logically independent and neither tautologous nor contradictory. This is a consequence of $\left(\mathrm{COM}^{\prime}\right)$ that will be of considerable importance as we go along. For the purpose of easy reference later on, let us label that requirement by (IN).

With recourse to $(\mathrm{COR})$ and $\left(\mathrm{COM}^{\prime}\right)$, correctness and completeness of the formalization of a text $\mathcal{T}$ composed of statements $A_{1}, \ldots, A_{n}$ can be syntactically defined as follows:

$\left(\mathrm{SYN}_{\mathcal{T}}\right)$ The formalization $\Phi$ of a text $\mathcal{T}$ is correct and complete iff $(\mathrm{COR})$ and $\left(\mathrm{COM}^{\prime}\right)$ are satisfied for all formalizations $\Phi_{1}, \ldots, \Phi_{n}$ of $A_{1}, \ldots, A_{n}$ and (COR) is satisfied for $\Phi$ of $\mathcal{T}$, such that $\Phi$ is a truth-function of $\Phi_{1}, \ldots, \Phi_{n}$.

$\Phi$ of $\mathcal{T}$ is the result of a truth-functional concatenation of $\Phi_{1}, \ldots, \Phi_{n}$, i.e. a well-formed expression consisting of $\Phi_{1}, \ldots, \Phi_{n}$ and truth-functional connectives. Demanding correctness for $\Phi$ of $\mathcal{T}$ suffices to identify a truth-function of $\Phi_{1}, \ldots, \Phi_{n}, n>1$, which shares its truth conditions with $\mathcal{T}$. This can be seen as follows: If $\Phi_{1}, \ldots, \Phi_{n}$ are (COR)-correct and $\left(\mathrm{COM}^{\prime}\right)$-complete for the component statements of $\mathcal{T}$-as required by $\left(\mathrm{SYN}_{\mathcal{T}}\right)$ - the realizations of $\Phi_{1}, \ldots, \Phi_{n}$ exclusively assign logically independent non-tautologous and non-contradictory expressions to the categorematic parts of these formulae. Against this background, it is guaranteed that any truth-functional concatenation $\Phi$ of $\Phi_{1}, \ldots, \Phi_{n}$ has the same truth conditions as its verbalization. ${ }^{29}$ Now, if $\Phi$ is correct for $\mathcal{T}, \mathcal{T}$ and the verbalization of $\Phi$ must be informally equivalent. For $\Phi$ formally implies itself and follows from itself, thus, according to (COR), $\mathcal{T}$ must both informally follow from and imply the verbalization of $\Phi$. Therefore, demanding correctness of $\Phi$ for $\mathcal{T}$ guarantees that $\Phi$ shares its truth conditions with $\mathcal{T}$.

It should be useful to sum up the interplay of the syntactic criteria of correctness and completeness when it comes to identifying the formalization which correctly and completely

29 This would not be guaranteed without (IN). If the formula $p$ is assigned a tautology as "It is raining or not raining" by its realization, the verbalization of $p$ does not coincide with $p$ as regards truth conditions: $p$ can be false, its verbalization cannot. 
exhibits a text $\mathcal{T}$ as a truth-function of its component statements $A_{1}, \ldots, A_{n}$. (COR) ensures that the realizations of $\Phi_{1}, \ldots, \Phi_{n}$ suit $A_{1}, \ldots, A_{n}$ (cf. p. 5). ( $\mathrm{COM}^{\prime}$ ) then properly tailors these realizations such that their constituents do not feature informal dependencies. Moreover, $\left(\mathrm{COM}^{\prime}\right)$ makes sure that all informal dependencies among $A_{1}, \ldots, A_{n}$ are formally captured by $\Phi_{1}, \ldots, \Phi_{n} .\left(\mathrm{SYN}_{\mathcal{T}}\right)$ finally identifies the proper truth-functional concatenation of $\Phi_{1}, \ldots, \Phi_{n}$.

As indicated above, completeness is only defined as a syntactic criterion in the literature. One reason for the lack of a semantic criterion of completeness may lie in the limitations of formal semantics introduced in order to formulate (TC) in a way that is equivalent to (COR). Section 2.2 has shown that truth conditions of formulae and statements are ordinarily seen to be comparable only if interpretations of formulae are eliminated from consideration if they are informally incoherent. This limitation of formal semantics not only gives rise to the problem of suitable interpretations (cf. Sect. 2.2), but moreover prohibits a feasible formulation of completeness by means of semantic criteria. To see this, consider the informally unsatisfiable statement $(\mathrm{k})$ along with formalization (15).

(k) Fury is a horse and no animal.

$$
\begin{gathered}
p \wedge \neg q \\
p: \text { Fury is a horse.; } q: \text { Fury is an animal. }
\end{gathered}
$$

Recall that in order to evaluate the correctness of (15) relative to (16), the informally unsuitable interpretation $\Im(p)=T, \Im(q)=F$ of (15) is to be eliminated from consideration. That means this limitation of formal semantics invalidates the only model of (15). The remaining interpretations all render (15) false. While (k) is informally unsatisfiable, (15) has no model among its suitable interpretations. Thus, (15) is not only (TC)-correct for (k), but would have to be seen as semantically complete as well. One of the intuitions behind demanding completeness of adequate formalizations consists in requiring informal dependencies among statements to be mirrored by formal dependencies. As (k) illustrates, this intuition is completely distorted if a formula as (15) which is non-contradictory according to classical logic — and therefore syntactically incomplete_-would have to be seen as a semantically complete formalization of an informally contradictory statement as (k). Apart from being counterintuitive, a semantic criterion of completeness that assesses completeness with exclusive recourse to suitable interpretations would not be equivalent to syntactic completeness in terms of $(\mathrm{COM})$ or $\left(\mathrm{COM}^{\prime}\right)$.

Completeness is commonly denied the status of a necessary condition of adequate formalization, because evaluating formalizations with respect to completeness blurs the distinction between logical formalization and semantic analysis. Maintaining this distinction amounts to one of the core desiderata of representatives of the traditional picture. Yet, eliminating interpretations $\mathfrak{\Im}$-in addition to generating the problem of suitable interpretations - already abolishes that distinction. In fact, it is impossible to keep logical formalization free from semantic analysis. If, consequently, the Tractarian view is adopted, completeness should be demanded of adequate formalizations such that informal dependencies can be accounted for by means of (classically understood) formal dependencies. This is straightforwardly accomplished by extending and modifying (TC) in a way that simultaneously captures correctness and completeness by semantic criteria and, moreover, solves the problem of suitable interpretations by simply eliminating that notion:

(TC') The formalization $\Phi$ of a statement $A$ is correct and complete iff relative to all interpretations $\Im$ of $\Phi, \Phi$ has the same conditions of truth and falsehood as $A$ has according to the informal judgement. 
The notions used in $\left(\mathrm{TC}^{\prime}\right)$ are straightforward: On the side of the formulae classical formal semantics is applied, on the side of the statements truth conditions are judged informally. The truth conditions to be evaluated are again generated by paraphrasing the formal interpretations with recourse to the realizations of the corresponding formulae (cf. Sect. 2.2).

$\left(\mathrm{TC}^{\prime}\right)$, on a par with $\left(\mathrm{COM}^{\prime}\right)$, does not demand to formalize a statement $A$ in a way that captures all informal inferential dependencies among $A$ and arbitrary other statements. Correspondingly, $\left(\mathrm{TC}^{\prime}\right)$ does not demand to reveal the inner structure of an isolated statement $A$. As per (TC'), all the formalizations (17)-(19) are correct and complete for

(1) There exists a mother.

$$
\begin{array}{rl}
p & p: \text { There exists a mother. } \\
\exists x F x & F: \ldots \text { is a mother } \\
\exists x(G x \wedge H x) & G: \ldots \text { is a woman } ; H: \ldots \text { has a child }
\end{array}
$$

However, if (1) is part of a complex statement, i.e. of a text $\mathcal{T}$ that features additional statements, informal dependencies among these further statements and (1) may well impose to reveal aspects of the inner structure of (1) in order to provide a complete formalization of $\mathcal{T}$. This is rendered explicit in the following semantic correctness and completeness criterion for the formalization of a text $\mathcal{T}$ composed of statements $A_{1}, \ldots, A_{n}$ :

$\left(\mathrm{TC}_{\mathcal{T}}^{\prime}\right)$ The formalization $\Phi$ of a text $\mathcal{T}$ is correct and complete iff $\left(\mathrm{TC}^{\prime}\right)$ is satisfied for all formalizations $\Phi_{1}, \ldots, \Phi_{n}$ of $A_{1}, \ldots, A_{n}$ and for $\Phi$ of $\mathcal{T}$, such that $\Phi$ is a truth-function of $\Phi_{1}, \ldots, \Phi_{n}$.

Whenever $\mathcal{T}$ consists of a single statement $A$ only, $\left(\mathrm{TC}_{\mathcal{T}}^{\prime}\right)$ is equivalent to $\left(\mathrm{TC}^{\prime}\right)$. In this sense, $\left(\mathrm{TC}_{\mathcal{T}}^{\prime}\right)$ generalizes $\left(\mathrm{TC}^{\prime}\right)$.

To illustrate how, based on the textual context, $\left(\mathrm{TC}_{\mathcal{T}}{ }_{\mathcal{T}}\right)$ determines to what extent the inner structure of a statement is to be revealed by a correct and complete formalization, consider the following exemplary conditionals in both of which (l) appears as antecedent:

(m) If there exists a mother, then somebody loves Labhaoise.

(n) If there exists a mother, then somebody is a woman.

The average English speaker will assess the statements "There exists a mother" and "Somebody loves Labhaoise" to be informally independent: It is possible that Labhaoise is loved without there being a mother, for instance when Labhaoise is a motherless divine being, and mothers may well exist without anybody loving Labhaoise. In contrast, it is informally excluded that something is a mother but no woman. Accordingly, $(\mathrm{m})$ is taken to be nontautologous, while (n) is seen as an informal tautology. That means, in order to formally represent the conditional $(\mathrm{m})$, its antecedent and consequent can be treated as (unanalyzed) independent propositions, whereas capturing the informal tautology (n) calls for an analysis of (1) in terms of (19). In this sense, (20) and (21) are $\left(\mathrm{TC}_{\mathcal{T}}^{\prime}\right)$-correct and -complete for (m) and (n), respectively.

$$
p \rightarrow q
$$

$p$ : There exists a mother.; $q$ : Somebody loves Labhaoise.

$$
\exists x(G x \wedge H x) \rightarrow \exists x G x
$$

$G$ : ...is a woman; $H:$. .has a child

Yet, it might happen that somebody deviates from the average understanding of (m) and considers that text to be an informal tautology after all. It could be argued that $(\mathrm{m})$ is informally tautologous, not due to semantic dependencies among "There exists a mother" and 
"Somebody loves Labhaoise", but due to a missing premise. Thus, (m) could be interpreted in terms of an enthymeme with, say, "Labhaoise is the goddess of motherhood" as missing premise. More specifically, the interpretation of (m) might be the following truth-function of "There exists a mother", "Labhaoise is the goddess of motherhood" and "Somebody loves Labhaoise":

(o) Whenever there exists a mother and Labhaoise is the goddess of motherhood and, if there exists a mother and Labhaoise is the goddess of motherhood, then somebody loves Labhaoise, then somebody loves Labhaoise.

Even relative to this interpretation the inner structure of (1) does not need to be formally exhibited in order to $\left(\mathrm{TC}_{\mathcal{T}}^{\prime}\right)$-correctly and -completely account for that text:

$$
p \wedge r \wedge(p \wedge r \rightarrow q) \rightarrow q
$$

$p$ : There exists a mother.; $q$ : Somebody loves Labhaoise.;

\section{$r$ : Labhaoise is the goddess of motherhood.}

Whenever correctness is separated from completeness, semantic criteria of adequate formalization face the problem of suitable interpretations. Such a separation prohibits a comparison of truth conditions of formulae and statements relative to all possible interpretations. Yet, notwithstanding the fact that the strive for complete formalizations motivates many famous formalization efforts, $\left(\mathrm{TC}_{\mathcal{T}}^{\prime}\right)$ is in disharmony with the common formalization practice. Commonly, (5), i.e. $p \vee q$ (cf. p. 8), is thought to be adequate for (c), i.e. "Cameron is a mother or Cameron is a woman", in virtue of realization (6). $\left(\mathrm{TC}_{\mathcal{T}}^{\prime}\right)$, however, identifies (5) as inadequate: $\Im(p)=W, \Im(q)=F$ renders (5) true, yet no consistent conditions of truth or falsehood correspond informally. $\left(\mathrm{TC}_{\mathcal{T}}^{\prime}\right)$ demands more than what is usually required by a criterion of correctness. If it is judged to be impossible for Cameron to be a mother, but no woman, this impossibility has to be captured by a formal contradiction. Generally, not only formal dependencies have to match informal ones, but also informal dependencies have to be mirrored by formal ones.

This requirement is met by formalizing (c) as follows:

$$
(p \wedge q) \vee q
$$

$p$ : Cameron has a child.; $q$ : Cameron is a woman.

(24) is formally equivalent to $q$, which exhibits that (c) has the same conditions of truth and falsehood as "Cameron is a woman". Moreover, the propositional variables of (24) are assigned to informally independent statements in (25). Therefore, paraphrasing the models and counter-models of (24) does not yield any informally incoherent conditions of truth and falsehood. Balancing these conditions reveals that the paraphrase of every model of (24) renders (c) true, while the paraphrase of every counter-model renders (c) false. Thus, (24) is a $\left(\mathrm{TC}_{\mathcal{T}}^{\prime}\right)$-correct and -complete formalization of (c).

As these exemplary formalizations demonstrate, adequately formalizing a statement in first-order logic in a way that satisfies $\left(\mathrm{TC}_{\mathcal{T}}^{\prime}\right.$ ) presupposes that the expressions assigned to the categorematic parts of a formula by its realization are logically independent and neither tautologous nor contradictory. The conditions of truth and falsehood generated by paraphrasing the interpretations of the formalization are required to consist of informally independent propositions. As $\left(\mathrm{COM}^{\prime}\right)$, thus, $\left(\mathrm{TC}_{\mathcal{T}}^{\prime}\right)$ demands maximal transparency with respect to informal dependencies among component statements of $\mathcal{T}$ and the elements of a formula's realization. As $\left(\mathrm{COM}^{\prime}\right),\left(\mathrm{TC}_{\mathcal{T}}^{\prime}\right)$ stipulates (IN) (cf. p. 17). Common formalization practice does not respect (IN). The reason is at hand: Logical formalization shall not be rendered 
dependent on a narrow conception of semantic analysis that explicates the conditions of truth and falsehood of statements with reference to informally independent propositions. If the adequacy of a formalization is judged with recourse to a criterion as $\left(\mathrm{TC}_{\mathcal{T}}^{\prime}\right)$, the amount of statements adequately formalizable within first-order logic such that no far reaching questions of semantic analysis arise, becomes much smaller than it is usually thought to be. Ever so often a semantic analysis of a statement that satisfies (IN) is simply not available in natural language. Nonetheless, the ambition to straightforwardly formalize a wide range of statements within first-order logic cannot be the guide to define criteria of adequate formalization. Rather, the fact that the theoretical underpinnings of the common formalization practice only seem to be reconstructible based on a distortion of formal semantics questions the rational foundation of that practice. Instead, one should come to realize that first-order formalization heavily depends on a conception of semantic analysis that exhibits complex statements as truth-functions of informally independent propositions.

\subsection{Adequacy}

Contrary to the traditional picture, we thus advance both correctness and completeness as necessary conditions of adequate formalization. As to the above considerations, this requirement can be spelled out either syntactically, i.e. in terms of $\left(\mathrm{SYN}_{\mathcal{T}}\right)$, or semantically, i.e. in terms of $\left(\mathrm{TC}_{\mathcal{T}}^{\prime}\right)$. As $\left(\mathrm{SYN}_{\mathcal{T}}\right)$ refers to $(\mathrm{COR})$ and $\left(\mathrm{COM}^{\prime}\right)$ whose application, contrary to the application of $\left(\mathrm{TC}^{\prime} \mathcal{T}\right)$, does not terminate and involves circularities (cf. Sect. 2.2) as well as in light of the simplicity of $\left(\mathrm{TC}_{\mathcal{T}}^{\prime}\right)$ we shall give preference to $\left(\mathrm{TC}_{\mathcal{T}}^{\prime}\right)$ in the following.

An informally valid argument is only $\left(\mathrm{TC}^{\prime}{ }_{\mathcal{T}}\right)$-correctly and -completely formalized by a formal implication and, analogously, an informally invalid argument must be captured by a material implication. Thus, contrary to the traditional picture that allows for non-tautologous formalizations of informally valid arguments, defining adequate formalization based on $\left(\mathrm{TC}_{\mathcal{T}}^{\prime}\right.$ ) paves the way to identify, i.e. to prove in a weak sense (cf. footnote 2 ), the validity as well as the invalidity of arguments. ${ }^{30}$

Furthermore, $\left(\mathrm{TC}_{\mathcal{T}}^{\prime}\right)$ prohibits unscrupulous formalization (cf. Sect. 2.3). The formalization of "Cameron is a mother" by $F a \wedge G a$, where $F$ represents ". . . is a mother", $G$ stands for ". . . is a woman" and $a$ for Cameron, does not satisfy $\left(\mathrm{TC}_{\mathcal{T}}^{\prime}\right)$, because according to formal semantics $F a \wedge G a$ is false, if the object assigned to $a$ is an element of $\Im(F)$ but not of $\Im(G)$, whereas Cameron's being a mother but no women is informally incoherent. Thus, $\left(\mathrm{TC}_{\mathcal{T}}^{\prime}\right)$ prevents an unscrupulous formalization of argument $(\mathrm{d})$. Nonetheless, $\left(\mathrm{TC}_{\mathcal{T}}^{\prime}\right)$ does not block all trivializations of logical formalization. If a formula $\Phi_{1}$ satisfies $\left(\mathrm{TC}_{\mathcal{T}}^{\prime}\right)$ with respect to a statement $A_{1}$ contained in a text $\mathcal{T}$, any formula $\Psi_{1}$ such that $\Phi_{1} \dashv \vdash \Psi_{1}$ satisfies $\left(\mathrm{TC}_{\mathcal{T}}^{\prime}\right.$ ) as well, provided that $\Psi_{1}$ meets (IN). In this sense, all components $\Phi_{1}, \ldots, \Phi_{n}$ of a formalization $\Phi$ of $\mathcal{T}$ are replaceable by equivalent components $\Psi_{1}, \ldots, \Psi_{n}$ to the effect that the resulting formalization $\Psi$ of $\mathcal{T}$ satisfies $\left(\mathrm{TC}_{\mathcal{T}}^{\prime}\right)$ iff $\Phi$ satisfies $\left(\mathrm{TC}_{\mathcal{T}}^{\prime}\right)$. Hence, $\left(\mathrm{TC}_{\mathcal{T}}^{\prime}\right)$ identifies sets of logically equivalent formulae as correct and complete formalizations of a given text. Accordingly, any formal tautology satisfying (IN) is a $\left(\mathrm{TC}_{\mathcal{T}}^{\prime}\right)$-correct and -complete formalization of any uncomposed informal tautology. If the adequacy of a formalization were only assessed with recourse to $\left(\mathrm{TC}_{\mathcal{T}}^{\prime}\right.$ ), it would be completely arbitrary which of $p \vee \neg p$

\footnotetext{
30 Massey (1975) pointed out that the invalidity of arguments can never be (in a weak sense) formally proven based on correctness criteria only. This problem of invalidity proofs is generally accepted (cf. e.g. Sainsbury (1991), pp. 68-69, 116-120). Representatives of the traditional picture hold that only the validity of arguments can be formally identified. Within the new picture of formalization presented here, both the validity and the invalidity of arguments can be (in a weak sense) formally proven (cf. Lampert and Baumgartner, unpublished).
} 
or $\neg(p \wedge \neg p)$ etc. should be propagated as adequate representation of a statement as "It is raining or it is not raining". The analog holds for formal and informal contradictions.

$\left(\mathrm{TC}_{\mathcal{T}}^{\prime}\right.$ ) thus must be complemented by surface considerations. Accordingly, we draw on surface similarity in order to identify adequate formulae within sets of equivalent formalizations that all satisfy $\left(\mathrm{TC}_{\mathcal{T}}^{\prime}\right)$. While the latter criterion often explicitly induces to depart from the surface of natural language, the similarity requirement amounts to a minimalization of the differences between logical formulae and statements. This strategy to complement $\left(\mathrm{TC}^{\prime} \mathcal{T}\right)$ yields the following — surface relativized—notion of adequate formalization:

(ADS) The formalization $\Phi$ of a text $\mathcal{T}$ is adequate iff $\left(\mathrm{TC}_{\mathcal{T}}^{\prime}\right.$ ) is satisfied and $\Phi$ is at least as similar to $\mathcal{T}$ as any other equivalent formula that satisfies $\left(\mathrm{TC}^{\prime} \mathcal{T}\right)$.

The similarity of the syntactic features of $\Phi$ and $\mathcal{T}$ is to be measured according to strategies (I) and (II) discussed in Section 2.3.

(ADS) does not identify one single formula as the adequate formalization of a text $\mathcal{T}$, but rather chooses formalizations that are maximally similar to $\mathcal{T}$ among every set of equivalent and $\left(\mathrm{TC}_{\mathcal{T}}^{\prime}\right)$-correct and -complete formalizations of $\mathcal{T}$. That means $p$ relative to " $p$ : Not all martians are green" may be an adequate formalization of "Not all martians are green", just as $\neg \forall x(F x \rightarrow G x)$ relative to " $F: \ldots$ is a martian; $G: \ldots$ is green". Among a set of equivalent formulae, including $p$ and $p \wedge(p \vee \neg p)$ and $p \wedge(q \leftrightarrow q)$, (ADS) picks $p$ as adequate representation of "Not all martians are green", because it is syntactically more similar to that statement than any other member of its equivalence set. $q$, however, relative to " $q$ : Not all martians are green" is no less adequate according to (ADS). Likewise, $\neg \forall x(F x \rightarrow G x)$ is more similar to "Not all martians are green" than, say, the equally $\left(\mathrm{TC}^{\prime} \mathcal{T}\right)$-correct and -complete $\neg \forall x(\neg \neg F x \rightarrow G x)$. By confining similarity considerations to a set of equivalent formulae the latter are rendered comparable as regards their syntactic similarity with the formalized statement.

\section{Against reflective equilibrium}

(ADS) - as the criteria of correctness and completeness - crucially rests on the notion of informal validity. As anticipated on page 5 above, the dependency of adequate formalization on informal validity raises the question as to how logic can serve as a means to evaluate the validity or invalidity of arguments. How could a formalism ever identify fallacies and correct an informal judgement in view of the fact that an adequate implementation of the formalism presupposes that informal judgement? Moreover, what about the usefulness of relativizing the adequacy of formalizations to a notion as vague as informal validity?

Ordinarily, such as to evade this problem, the relationship between natural and formal languages is conceived in terms of a so-called reflective equilibrium, which derives from Rawls (1980) and Goodman (1983). ${ }^{31}$ According to this conception, informal validity is the gauge that measures the quality of formal definitions of what valid derivations are and formal validity systemizes and regulates its informal counterpart. Formal validity analyzes and theoretically represents informal reasoning, while informal (in)validity assessments may be reversed for reasons of systematics, conceptual simplicity, or incompatibility with accepted background theories. Logical formalization and its reversal, the transformation of formulae

31 Cf. e.g. Hoyningen-Huene (1998), pp. 155 et sqq., or Brun (2004), pp. 76 et sqq., Löffler (2006). For the original context, in which the notion of a reflective equilibrium has arisen, see Rawls (1980), p. 20, and Goodman (1983), pp. 63 et sqq. 
into statements called verbalization, are localized at the core of this equilibrium as they mediate between the implementation of informal and formal validity.

Apart from the difficulties arising from the attempt to ground a methodology of formalization on the notion of a reflective equilibrium in a sharp and non-circular way, there is one main problem with this conception. As the notion of informal validity is presupposed by the criteria of adequate formalization, such criteria would be deprived of their status as necessary conditions for the adequacy of a formalization given that informal reasoning always risks to be revised by formal constraints. Thus, as an immediate consequence of a methodology of formalization embedded in equilibrium considerations, criteria of adequate formalization become mere rules of thumb that are sometimes resorted to upon formalizing natural language and sometimes neglected. Criteria are no criteria if they are open for discussion.

Endorsing the ars iudicandi conception of logic by basing formalization on rules of thumb, in our view, is too high a price to pay. Rather, we prefer to abstain from claiming that logic provides the means to prove the (in)validity of colloquial arguments in a strong sense, according to which proving an argument amounts to formally evaluating whether it is valid or not. Formalizing an argument does not promote a proof in this strong sense, but, rather, an explication of the argument. ${ }^{32}$ A formalization of an argument replaces an ambiguous and mistakable expression by an unambiguous and unmistakable formula that transparently represents the formal structure on which the argument is based. Formalization, thus, is a means to explicate informal reasoning which does not rest on criteria. According to this view, formalizations are not and will never be the result of a formal, effective formalization procedure, because formalizing a natural language text $\mathcal{T}$ crucially rests on an informal understanding of $\mathcal{T}$ and not simply on its surface grammar.

Admitting the impossibility to prove the validity or invalidity of arguments in a strong sense moreover answers the question as to why we have analyzed adequate formalization with recourse to a notion as vague as informal validity. Our analysis of adequate formalization does not impose there to be one adequate formula for an argument — there may be many or none at all. Rather, a formalization expresses or explicates informal reasoning relative to an interpretation of a text $\mathcal{T}$. Informal judgements about inferential dependencies among $\mathcal{T}$ 's component statements $A_{1}, \ldots, A_{n}$ may vary and, accordingly, corresponding formalizations differ. Nonetheless, every formalization unambiguously expresses informal judgements. We do not claim to provide criteria that ultimately identify a specific formula as the one and only adequate formalization of a given statement. (ADS) determines formalizations to be adequate relative to a decomposition of $\mathcal{T}$ and informal judgements concerning the conditions of truth and falsehood of $A_{1}, \ldots, A_{n}$, i.e. relative to an interpretation of $\mathcal{T}$.

Abandoning an ars iudicandi conception of logic in favor of an ars explicandi has a number of important consequences. First, whenever internal dependencies among the statements in a text or the truth conditions of these statements cannot be judged informally, there is no criterion that would determine the adequacy of a respective formalization. A logical formalism cannot clarify what is informally indeterminate. Second, whenever there is a conflict between informal considerations and corresponding formalizations there do not exist two feasible sources of error-defectiveness of the informal judgement or inadequacy of the formalization-but only one: In case of conflict it is always the formalization that is in need of revision. Third, a logical formalism can never revise informal reasoning. Whenever an argument is informally claimed to be valid, it cannot be identified as a fallacy. There are no

\footnotetext{
32 We do not use the term 'explication' in Carnap's sense (cf. Carnap (1971), Subsect. 2-3). While for Carnap explicandum and explicans may differ in meaning or truth conditions, respectively, $\left(\mathrm{TC}^{\prime} \mathcal{T}\right)$ determines that a formal explicans coincides with its informal explicandum with regard to truth conditions. In virtue of its syntax alone the formalization explicates the informally assessed truth conditions of the formalized text.
} 
informal fallacies. What is ordinarily called a 'fallacy' or a 'paradox' is not mistaken informal reasoning, but rather a misunderstanding of informal arguments expressed by inadequate formalizations.

To somebody professing the traditional ars iudicandi conception of logic these consequences, most likely, seem counterintuitive. Presupposing clarity about informal inferential dependencies among components of a formalized text, however, is not a peculiarity of the new picture of adequate formalization. As Sect. 2.2 has shown, such transparency is also a precondition of applying the traditional correctness criterion. According to both the traditional and the new picture, the adequacy of formalizations cannot be judged without explicit informal judgements about the whole argumentative context. Yet, whoever sees logic as an ars iudicandi and, thus, claims that formalizing an argument may decide on its informal validity, tacitly presupposes that the adequacy of a formalization is independent of a prior judgement as to the validity of the corresponding argument. If formalizations are brought to bear in order to evaluate the validity of an argument, the adequacy of these formalizations must be established without drawing on an informal validity judgement. However, the literature developing the traditional picture of formalization does not even provide sketches of an adequacy criterion that would be independent of informal validity judgements or informal assessments of truth conditions of the constituents of formalized arguments. As soon as one comes to realize that, first, the adequacy of formalizations must be justified if anything interesting is to be gained from formalizing arguments and, second, that such a justification inescapably hinges on clarity about the informal validity of the argument and the truth conditions of its premises and conclusion, abstaining from the traditional ars iudicandi conception of logic appears as the only remaining consequence. One of the main goals of this paper, accordingly, has been to show that intuitions to the contrary, regardless of their prevalence, are ill-founded.

Acknowledgements We would like to thank Georg Brun for intensive discussions about earlier drafts of this paper as well as two anonymous referees for helpful comments. Timm Lampert is partially supported by the Swiss National science foundation.

\section{References}

Blau, U. (1977). Die dreiwertige Logik der Sprache. Berlin: de Gruyter.

Brun, G. (2004). Die richtige Formel. Philosophische Probleme der logischen Formalisierung. Frankfurt a.M.: Ontos.

Carnap, R. (1971). The logical foundations of probability. London: Routledge.

Chomsky, N. (1977). Essays on form and interpretation. Amsterdam: North-Holland.

Chomsky, N. (1986). Knowledge of language. New York: Praeger.

Davidson, D. (1967). The logical form of action sentences. In N. Rescher (Ed.), The logic of decision and action (pp. 81-95). Pittsburgh: University of Pittsburgh Press.

Davidson, D. (1980). Essays on actions and events. Oxford: Clarendon Press.

Davidson, D. (1984). Inquiries into truth and interpretation. Oxford: Oxford University Press.

Epstein, R. L. (1990). The semantic foundations of logic: Propositional logic. Dordrecht: Kluwer.

Epstein, R. L. (1994). The semantic foundations of logic: Predicate logic. Oxford: Oxford University Press.

Goodman, N. (1983). Fact, fiction, and forecast (4th ed.). Cambridge: Harvard University Press.

Hoyningen-Huene, P. (1998). Formale Logik. Stuttgart: Reclam.

Jackson, B. (2007). Beyond logical form. Philosophical Studies, 132, 347-380.

Lampert, T. (2000). Wittgensteins Physikalismus. Paderborn: Mentis.

Lampert, T. (2006). Explaining formulae of first order logic. Ruch Filozoficzny, LXIII.3, 459-480.

Lampert, T., \& Baumgartner, M. The unity of logical form. (unpublished).

Link, G. (1979). Montague-Grammatik. München: Wilhelm Fink Verlag. 
Löffler, W. (2006). Spielt die rhethorische Qualität von Argumenten eine Rolle bei deren logischer Analyse? Überlegungen zum Verhältnis von Argumentationstheorie und formaler Logik. In G. Kreuzbauer \& G. Dorn (Eds.), Argumentation in Theorie und Praxis (Salzburger Beiträge zu Rhetorik und Argumentationstheorie, Band 1) (pp. 115-130). Wien: LIT.

Massey, G. J. (1975). Are there any good arguments that bad arguments are bad? Philosophy in Context 4, $61-77$.

Montague, R. (1974a). Quantification in ordinary language. In R. H. Thomason (Ed.), Formal philosophy: Selected papers of Richard Montague (pp. 247-270). New Haven: Yale University Press.

Montague, R. (1974b). Universal grammar. In R. H. Thomason (Ed.), Formal philosophy: Selected papers of Richard Montague (pp. 222-246). New Haven: Yale University Press.

Montague, R., \& Thomason, R. H. (Ed.) (1974). Formal philosophy: Selected papers of Richard Montague. New Haven: Yale University Press.

Neale, S. (1994). Logical form and LF. In C. P. Otero (Ed.), Noam Chomsky. Critical assessments. (pp. 788-838). London: Routledge.

Quine, W. v. O. (1953). Mr. Strawson on logical theory. Mind, 62, 433-451.

Rawls, J. (1980). A theory of justice. Oxford: Oxford University Press.

Read, S. (1994). Formal and material consequence. Journal of Philosophical Logic, 23, 247-265.

Sainsbury, R. M. (1991). Logical forms (2nd ed.). Oxford: Blackwell.

Strawson, P. F. (1952). Introduction to logical theory. London: Methuen.

Wittgenstein, L. (1995). Tractatus logico-philosophicus. Frankfurt a. M.: Suhrkamp. 